\title{
Modeling and experiment on tapered hollow waveguide multiplexer for multi- wavelength VCSEL array
}

\author{
Akihiro Imamura $^{a)}$, Naoto Kitabayashi, and Fumio Koyama \\ Microsystem Research Center, Tokyo Institute of Technology, \\ 4259-R2-22 Nagatsuda, Midori-ku, Yokohama 226-8503, Japan \\ a)imamura.a.aa@m.titech.ac.jp
}

\begin{abstract}
A tapered hollow waveguide multiplexer is proposed to combine the output of a GaInAs/GaAs multi-wavelength VCSEL array for coupling into a multi-mode fiber. The design of the proposed hollow waveguide is presented based on ray optics. We demonstrate multiplexing of 4-channel output of a GaInAs/GaAs VCSEL array formed on a patterned substrate for coupling into a multi-mode fiber. The proposed hollow waveguide multiplexer is useful for realizing compact and lowcost WDM transceiver with a multi-wavelength VCSEL array for high capacity short reach optical networks.
\end{abstract}

Keywords: VCSEL, hollow waveguide, WDM, laser array

Classification: Photonics devices, circuits, and systems

\section{References}

[1] F. Koyama, T. Mukaihara, Y. Hayashi, N. Ohnoki, N. Hatori, and K. Iga, "Two-dimensional multiwavelength surface emitting laser arrays fabricated by nonplanar MOCVD," Electron. Lett., vol. 30, no. 23, pp. 19471948, 1994.

[2] H. Saito, I. Ogura, and Y. Sugimoto, "Uniform CW operation of multiplewavelength vertical-cavity surface-emitting lasers fabricated by mask molecular beam epitaxy," IEEE Photon. Technol. Lett., vol. 8, pp. 11181120, 1996.

[3] J.-H. Shin and B.-S. Yoo, "Fabrication method for multiple wavelength vertical cavity emitter arrays by SiN layer thickness control," IEEE Photon. Technol. Lett., vol. 11, pp. 509-511, 1999.

[4] D. L. Huffaker and D. G. Deppe, "Multiwavelength, densely-packed 2x2 vertical cavity surface emitting laser array fabricated using selective oxidation," IEEE Photon. Technol. Lett., vol. 8, pp. 858-860, 1996.

[5] S.-Y. Hu, J. Ko, E. R. Hegblom, and L. A. Coldren, "Multimode WDM optical data links with monolithically integrated multiple-channel VCSEL and photodetector arrays," IEEE J. Quantum Electron., vol. 34, pp. 1403-1414, 1998.

[6] A. Fiore, Y. A. Akulova, J. Ko, E. R. Hegblom, and L. A. Coldren, "Postgrowth tuning of semiconductor vertical cavities for multiple-wavelength laser arrays," IEEE J. Quantum Electron., vol. 35, pp. 616-623, 1999. 
[7] M. Arai, T. Kondo, A. Onumura, A. Matsutani, T. Miyamoto, and F. Koyama, "Multiple-Wavelength GaInAs/GaAs Vertical Cavity Surface Emitting Laser Array With Extended Wavelength Span," IEEE J. Sel. Topics Quantum Electron., vol. 9, no. 5, pp. 1367-1373, 2004.

[8] Y. G. Ju, D. Lofgreen, A. Fiore, S. Y. Hu, E. Hegblom, D. Louderback, O. Sjolund, A. Huntington, and L. A. Coldren, "Densely packed pie shaped vertical-cavity surface-emitting laser array incorporating a tapered onedimensional wet oxidation," IEEE Photon. Technol. Lett., vol. 12, no. 5, pp. 462-464, 2000.

[9] B. E. Lemoff, M. E. Ali, G. Panotopoulos, E. Groot, G. M. Flower, G. H. Rankin, A. J. Schmit, K. D. Djordjev, M. R. T. Tan, A. Tandon, W. Gong, R. P. Tella, B. Law, L.-K. Chia, and D. W. Dolfi, "Demonstration of a compact low-power 250-Gb/s parallel-WDM optical interconnect," IEEE Photon. Technol. Lett., vol. 17, no. 1, pp. 220-222, 2005.

[10] T. Miura, F. Koyama, Y. Aoki, A. Matsutani, and K. Iga, "Hollow optical waveguide for temperature-insensitive photonic integrated circuits," Jpn. J. Appl. Phys., vol. 40, no. 7A, pp. L688-L690, 2001.

[11] N. Kitabayashi, A. Matsutani, and F. Koyama, "Multi-wavelength vertical cavity surface emitting laser array and tapered coupler on patterned substrate," International Symposium Contemporary Photonics Technology, CPT 200\%, G-7, pp. 79-80, Jan. 10-12, 2007.

\section{Introduction}

Multi-wavelength vertical cavity surface emitting laser (VCSEL) array is a good candidate for wavelength division multiplexing (WDM) short reach systems to increase the data capacity of local area networks (LAN), optical interconnects and so on. Various types of multi-wavelength VCSELs have been demonstrated $[1,2,3,4,5,6,7]$. We realized a multi-wavelength VCSEL array exhibiting a wavelength span of $190 \mathrm{~nm}$ using MOCVD on patterned substrates [8]. In order to realize WDM transceivers based on multi-wavelength VCSEL arrays, however, a low-cost and compact multiplexer of each VCSEL output is necessary. So far either direct coupling or use of dielectric multilayer filters has been demonstrated $[8,9]$. We have developed hollow waveguide optical devices exhibiting various unique features of temperature insensitivity and large tunability and so on [10].

In this paper, we propose and demonstrate a tapered hollow waveguide multiplexer for coupling the output from multi-wavelength VCSEL array into a multi-mode fiber.

\section{Device structure and modeling}

Our proposed tapered hollow waveguide multiplexer is schematically shown in Fig. 1. The structure consists of two high-reflectivity mirrors. The core is air and a multi-wavelength VCSEL array is integrated on the bottom mirror in the figure. Each output from VCSEL is radiated vertically from the bottom mirror side. An upper mirror and bottom mirror are placed with a taper angle $\theta$. The traveling of the output along the hollow waveguide is analyzed 
based on simple ray optics as shown in Fig. 1. After multiple reflections, the traveling angle $\phi$ of the output, which is defined from the bottom mirror surface, is simply expressed by

$$
\phi=2 \times n \times \theta
$$

where $\mathrm{n}$ is the number of reflections. Therefore the propagation direction of the output from each VCSEL is converted to a horizontal direction. The output from the tapered hollow waveguide multiplexer can be coupled into a multimode fiber when the traveling angle can be within the numerical aperture NA of the fiber. Thus, a simple and low-cost multiplexer can be realized without losing the advantages of small foot print of VCSEL arrays.

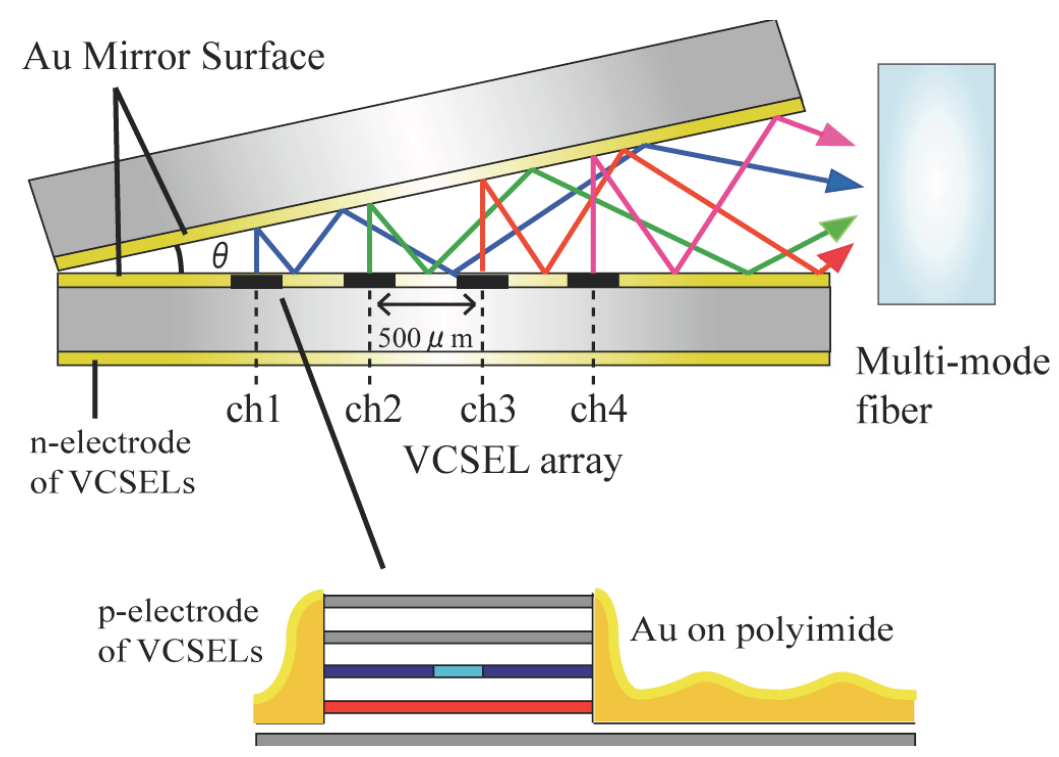

Fig. 1. Schematic of tapered hollow waveguide multiplexer with multi-wavelength VCSEL array.

Figure 2 shows the calculated ray trace for 4-channel-VCSEL array with a pitch of $20 \mu \mathrm{m}$. The traveling angle of ray-trace propagation depending on the VCSEL position in the multiplexer can be within the NA of a multi-mode fiber at the end of the hollow waveguide coupler. The output air-core size is assumed to be $50 \mu \mathrm{m}$ for matching with a $50 \mu \mathrm{m}$-multi-mode fiber. The opposite side of the upper mirror is connected to the bottom mirror. We discuss the scalability of the tapered hollow waveguide multiplexer. We calculated the output angle of the ray trace from each VCSEL element and the minimum length of the multiplexer for various taper angles $\theta$ so that the output angle $\phi$ can be within the $\mathrm{NA}=0.35$ of a muti-mode fiber. Figure 3 (a) shows the calculated multiplexer device length to satisfy the condition that the output of all the VCSELs can be coupled into the multi-mode fiber. Assuming that the pitch of a VCSEL array is $20 \mu \mathrm{m}$, we obtain the maximum number channel of the VCSEL array as shown in Fig. 3 (b). We also calculated the insertion loss by counting the number of reflections in the tapered hollow waveguide. The insertion loss is dependent on the position of each VCSEL. 
Figure 3 (c) shows the maximum and minimum insertion losses as a function of the taper angle where the reflectivity of the two mirrors is $98 \%$. We expect a compact $(<0.5 \mathrm{~mm})$ and low insertion loss $(<1 \mathrm{~dB})$ multiplexer for a 10 channel-VCSEL array from the results.

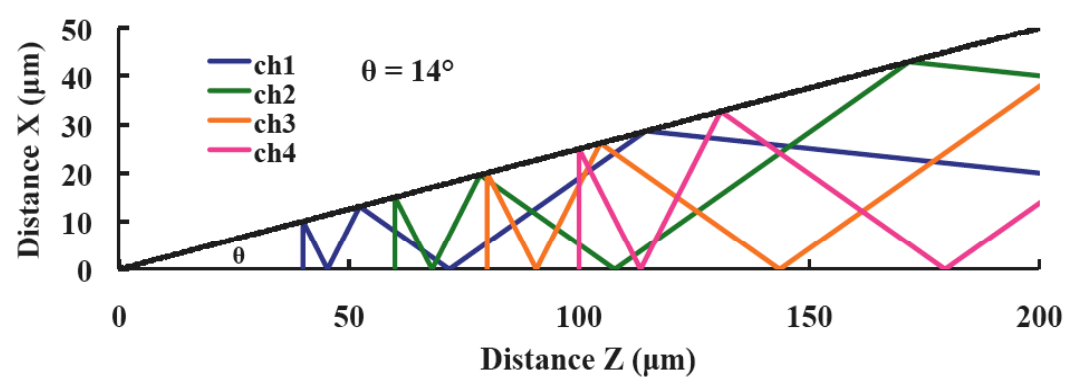

Fig. 2. Ray trace of a tapered hollow waveguide multiplexer with 4ch-VCSEL array.

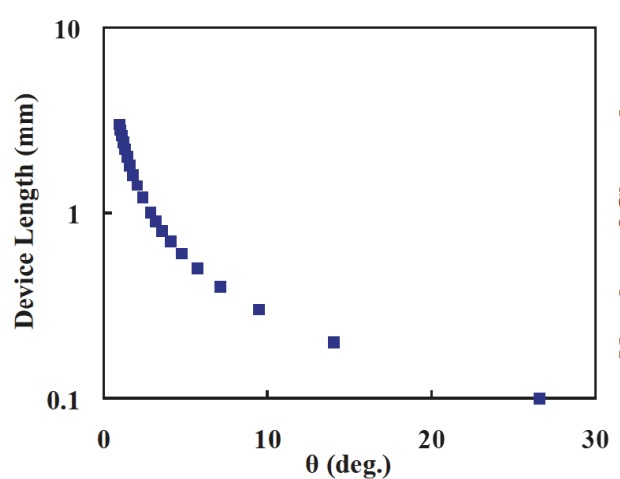

(a)

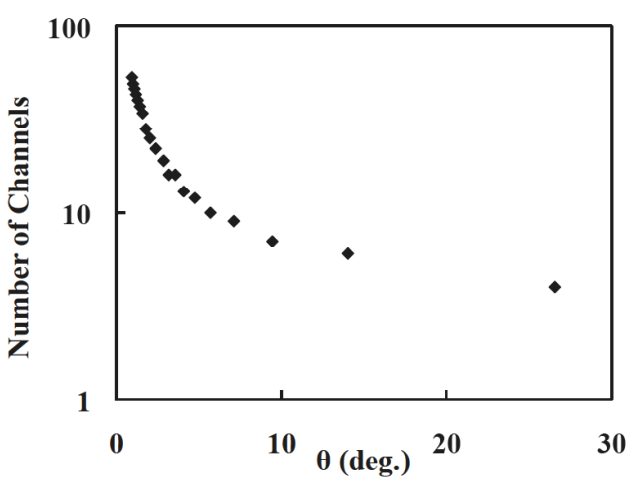

(b)

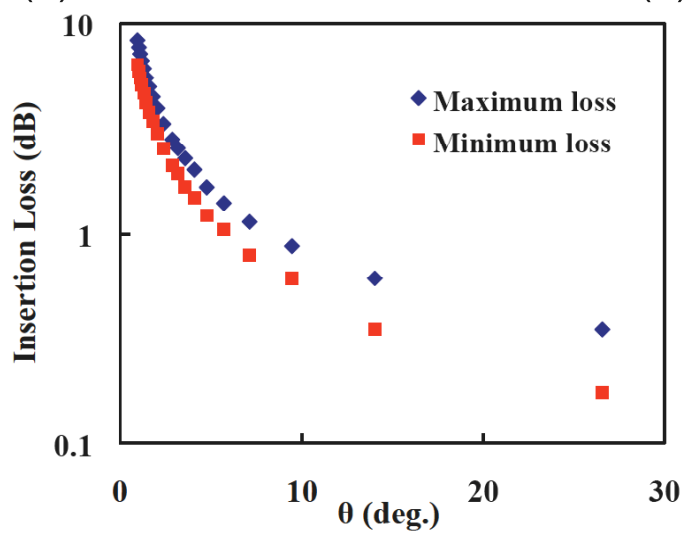

(c)

Fig. 3. (a) Calculated multiplexer length, (b) the maximum number of channels and (c) insertion loss as a function of a taper angle $\theta$ of a tapered hollow waveguide.

\section{Experiment}

We fabricated the tapered hollow waveguide multiplexer with multiwavelength VCSEL array on a patterned substrate [11]. A VCSEL array was fabricated using MOCVD on a patterned substrate, consisting of 22pair- 
$\mathrm{Al}_{0.85} \mathrm{Ga}_{0.15} \mathrm{As} / \mathrm{GaAs}$ p-DBR for the top mirror, 35.5pair- $\mathrm{Al}_{0.85} \mathrm{Ga}_{0.15} \mathrm{As} /$ GaAs n-DBR for the bottom mirror, an active region of 8nm-thick $\mathrm{Ga}_{0.67} \mathrm{In}_{0.33}$ As/GaAs 3 quantum wells (QWs) with $30 \mathrm{~nm}$-thick GaAs barriers. In order to use a VCSEL array chip as a bottom mirror for making a slab hollow waveguide, Au was deposited except a $30 \mu \mathrm{m}$ square output window. An upper mirror was also prepared by depositing a $\mathrm{Au}$ on a GaAs substrate and the length is $2 \mathrm{~mm}$. A four channel multi-mode VCSEL array was used for this experiment. The pitch of each element of the array is $500 \mu \mathrm{m}$. Au was deposited on the surface of the device and thus all the VCSEL was driven simultaneously at $8.6 \mathrm{~mA}$ per device.

The air core thickness at an output side and tapered angle was precisely controlled by a PZT actuator and a micro rotation stage. Figure 3 (a) shows the near field pattern at the output of a tapered hollow waveguide multiplexer when the output air core is $50 \mu \mathrm{m}$ and the taper angle is 1.4 degree. With this condition, the combined output from the VCSEL array was directly coupled with a $50 \mu \mathrm{m}$-core multi-mode fiber. Figure 3 (b) shows the spectra of multiplexed four channel output coupled into a fiber. The insertion loss was $35 \mathrm{~dB}$. This large insertion loss is primarily due to poor reflectivity of the bottom mirror and lateral diffraction in the slab hollow waveguide since no lateral confinement structure is formed in this experiment. We estimated the average reflectivity of the used mirror surface by ray trace analysis and found that it would be deteriorated from a theoretical Au reflectivity of $98 \%$ to $80 \%$. We believe this is due to rough polyimide surface between each VCSEL and mesa steps. By introducing the trench structure for each VCSEL mesa and a highly reflective DBR, of which theoretical reflectivity is over $99.9 \%$ for a hollow waveguide, the insertion loss will be reduced below $1 \mathrm{~dB}$.

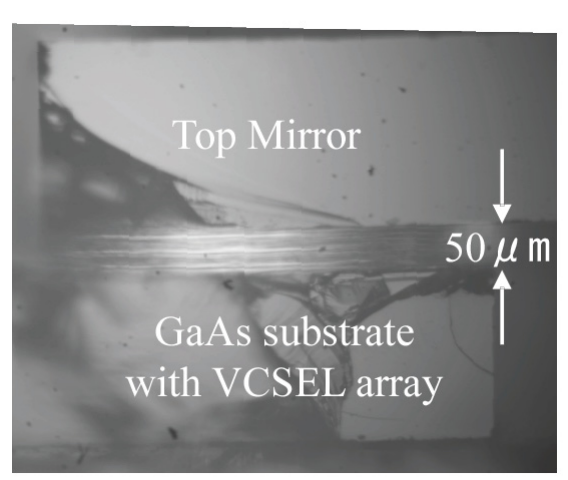

(a)

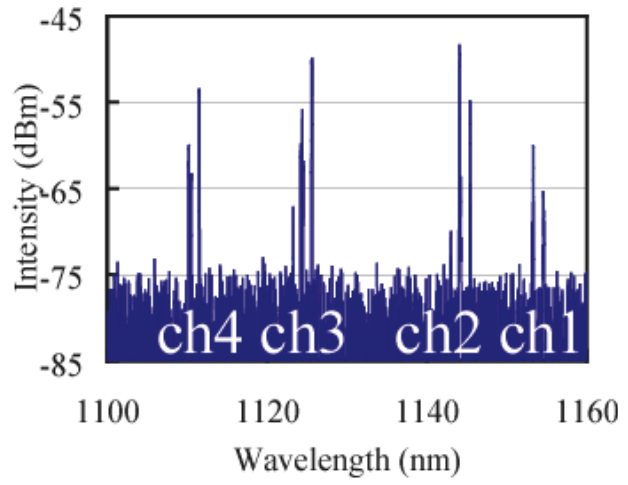

(b)

Fig. 4. (a) Near-field pattern at the output of a tapered hollow waveguide multiplexer and (b) spectra of the multiplexed four channel VCSEL output coupled into a multi-mode fiber. 


\section{Conclusion}

We proposed a compact tapered hollow waveguide muti-mode multiplexer to combine the output from a multi-wavelength VCSEL array for coupling into a multi-mode fiber. We demonstrated the multiplexing of four channel VCSEL output into a $50 \mu \mathrm{m}$ core multi-mode fiber. The present large insertion loss in this experiment will be improved by increasing the mirror reflectivity of the hollow waveguide. The proposed hollow waveguide multiplexer would be useful for realizing compact, simple and low-cost WDM transceivers for short reach applications.

\section{Acknowledgment}

This work was supported by National Institute of Information and Communication Technology. 